\title{
High Speed Soldering Using Laser Printing for the Assembly of Electronic Components
}

\author{
M. Makrygianni ${ }^{1}$, E. Margariti ${ }^{+1}$, K. Andritsos ${ }^{1}$, D. Reppas ${ }^{2}$, F. Zacharatos ${ }^{1}$, N. Oikonomidis ${ }^{2}$, \\ Ch. Spandonidis ${ }^{2}$, I. Zergioti ${ }^{* 1}$ \\ ${ }^{1}$ National Technical University of Athens, Physics Department, Zografou, 15780, Athens, Greece \\ ${ }^{2}$ Prisma Electronics SA, Alexandroupolis, 68 132, Greece \\ *Corresponding author's e-mail: zergioti@,central.ntua.gr \\ ${ }^{+}$Present address: Institute of Photonics, Department of Physics, University of Strathclyde, Technol- \\ ogy and Innovation Centre, Glasgow G1 1RD, United Kingdom
}

\begin{abstract}
Recent advancements in printed circuit board (PCB) assembly and IC packaging technology demand high resolution for ultra-fine pitch components $(<0.3 \mathrm{~mm}$ and $<60 \mu \mathrm{m}$ respectively), high throughput and compatibility with flexible substrates. These challenges are poorly met by the conventional deposition techniques (i.e. stencil printing), thus the need for a digital and high-resolution deposition technology is essential. In this context, Laser-Induced Forward Transfer (LIFT) can offer an excellent alternative: it is fully compatible with a wide variety of materials, it is environmentally friendly and offers versatile control over the printed volume with high throughput. In this work, a novel process for LIFT printing lead-free, jettable solder paste (powder size 15-25 $\mu \mathrm{m}$ ) is reported. A side-view imaging configuration is employed by coupling a LIFT setup with a high-speed camera for the real-time visualization of the ejection process. The experimental data from the captured videos are analyzed to determine morphological aspects. Transfers are achieved with diameters ranging from 50 to $150 \mu \mathrm{m}$ and printed volumes down to sub-nl. The fabrication of patterns, such as lines, on the PCB pads has been accomplished by scanning the laser beam over the donor surface (with 1 $\mathrm{m} / \mathrm{s}$ scanning speed), creating consecutive jets which form overlapping droplets.
\end{abstract}

DOI: $10.2961 / \mathrm{jlmn} .2020 .03 .2008$

Keywords: solder paste printing, laser-induced forward transfer, digital printing, chip bonding, microelectronics

\section{Introduction}

Flip-chip (FC) bonding of electronic and optoelectronic components is a key technology in system packaging and integration offering major advantages over the most commonly used wire bonding technique, such as shorter interconnect distance, high packaging density, smaller parasitic resistance, and improved device performance [1]. At the same time, current trends in microelectronics are driven by downscaling and increased complexity of functionality onto integrated circuit chips. With decreasing chip size, the density of interconnects increases and this demands advanced high-resolution interconnection technologies. Moreover, recently, large-area flexible electronics on low cost polymer substrates are attracting increasing interest [2], hence the compatibility of the interconnection technology with the carrier substrate has become more relevant than ever. Various technologies exist for the deposition of conductive adhesives [3], among which dispensing and stencil printing are most commonly used in traditional electronics manufacturing. Despite their maturity and wide usage, these two printing processes have inherent disadvantages that ensure that reliable high-resolution deposition of conductive adhesives required for miniaturized interconnections on rigid as well as flexible substrates remains a major challenge [4]. In the case of dispensing, the limiting factor is the nozzle dimension; even state-of-the-art dispensing systems cannot print sub-200 $\mu \mathrm{m}$ conductive adhesive dots in a consistent manner. Although recent research advances in stencil printing have demonstrated that sub-100 $\mu \mathrm{m}$ resolution can be attained [4], it is an inherently inflexible process that requires masks, and works in contact mode. Masks are subject to wear and deformation, making the printing of small interconnections distributed over large areas challenging. The need for a mask-less, high resolution deposition technique for the achievement of ultra-fine pitch interconnects is now essential. Among the various digital deposition and printing technologies, laser printing relying on the Laser Induced Forward Transfer (LIFT) technique stands out: LIFT technique was first introduced in the early 70s $[5,6]$ and was further investigated by Bohandy et al. [7] for the laser printing of copper patterns on silicon substrates. LIFT has been progressing ever since and recently has been used for the fabrication of flexible circuits, organic devices and sensors [8]. Compared to other deposition and printing techniques, where multiple, costly and time-consuming processes are often needed, LIFT offers the advantage of highly localised $(20 \mu \mathrm{m}$ resolution 
in liquid phase and $<10 \mu \mathrm{m}$ in solid phase LIFT) and direct deposition of features with minimum usage of solvents and waste fluids ( $\mu$ l volumes). In addition, the technique is not limited by the fluid properties, as in the case of ink-jet printing, since it can print materials with viscosities ranging from 1 up to more than 200,000 cP [9], it is a Computerized Numerical Controlled (CNC) digital technology which doesn't require complex mask patterns and most importantly is compatible with a wide range of substrate materials. The technique involves a transparent to the laser wavelength donor substrate, which is coated with the material of interest and is placed in close proximity to a receiver substrate. Upon irradiation, a part of the material is driven towards the receiver substrate. A laser pulse irradiating the back side of the donor substrate induces a rapid thermal increase at the interface of the donor substrate and the coated material creating a vapour bubble that propels the material towards the receiver substrate [10]. Previous works on laser transfer techniques have reported the printing of functional electronic circuits embedded on electronic devices as well as the transfer of parts of active device components [11]. LIFT and laser sintering techniques have been combined to print conductive silver nanoparticle (Ag NP) ink and paste lines on silicon and polyimide substrates [12]. The authors have successfully demonstrated the printing of fine features of $\mathrm{Ag}$ nanoink (viscosity $<100 \mathrm{cP}$ ) [13] for the fabrication of electrode patterns with dimensions of the order of $100 \mu \mathrm{m}$ and the controllable and reproducible printing of microdots of Ag nanopaste (viscosity $>100 \mathrm{kcP}$ ) on planar surfaces or even on vertical sidewalls [14,15] for high electrical conductivity interconnections.

Depending on the ink viscosity, different types of voxel behaviour during ejection are observed [16]. An intermediated viscosity ranges from $1 \mathrm{kcP}$ up to $1000 \mathrm{kcP}$ will allow transfer of integer voxels that retain the shape of the beam profile and "congruent" transfer is achieved. Congruent-dot transfer is the most useful for printing high-aspect-ratio dots, and the highest aspect ratios were achieved. Recently, S. A. Mathews et al., demonstrated the specific application of LIFT for solder paste printing [17] with resolution down to $25 \mu \mathrm{m}$, using reduced powder sized solder pastes (type 6 and 7) and donor/receiver gap smaller than $25 \mu \mathrm{m}$. In a study by K. S. Kaur et al. [18], LIFT was used to print type 5 solder paste pixels $(300 \mu \mathrm{m}$ x $300 \mu \mathrm{m})$, keeping a $35 \mu \mathrm{m}$ donor/receiver gap for light emitting diodes (LEDs) applications. However, in this work we will further extend the LIFT of solder bumps at ultrafine pitches $(50-150 \mu \mathrm{m})$, by demonstrating a high throughput and reproducible digital process, capable of achieving more than 100 kbumps per second (with high repetition rate lasers), offering substantial yield and speed improvements. In particular, we will investigate the effect of donor/receiver gap and more specifically for gaps larger than $100 \mu \mathrm{m}$ which are desirable for industrial applications, and the effect of laser flu- ence on the quality of both the resulting jets and the printed bumps. The outcomes of this investigation further substantiate the capability of to address challenges in soldering related to uniformity and reproducibility, speed and yield.

\section{Materials and methods}

\subsection{Experimental Setup}

The experimental setup used for these experiments consisted of two main subsystems, the laser printing configuration and the highspeed imaging system. More specifically, a ns DPSS Nd:YAG laser was used, operating at $532 \mathrm{~nm}$, with $20 \mathrm{~W}$ maximum output power, and $500 \mathrm{kHz}$ maximum repetition rate. More specifically, for repetition rates 10 and $100 \mathrm{kHz}$ used in this study, pulse duration was 20 and $40 \mathrm{~ns}$, respectively. The laser spot size for all experiments was 90 $\mu \mathrm{m}$, where laser spot size refers to the diameter of the laser spot at the donor/ carrier interface. The laser beam was scanned with speeds up to $3 \mathrm{~m} / \mathrm{sec}$, by using a galvanometric scanner and an f-Theta lens with a focal length of $170 \mathrm{~mm}$.

The highspeed imaging setup, consisted of a high-speed camera (Mini AX-100) coupled to the system, with maximum recording speed of this model was $540 \mathrm{kfps}$. In this work, a recording at $127 \mathrm{kfps}$ (one frame every $7.8 \mu \mathrm{s}$ ) was found to be adequate for the study of the jet propagation. Experimental setup is described in more detail in [19]

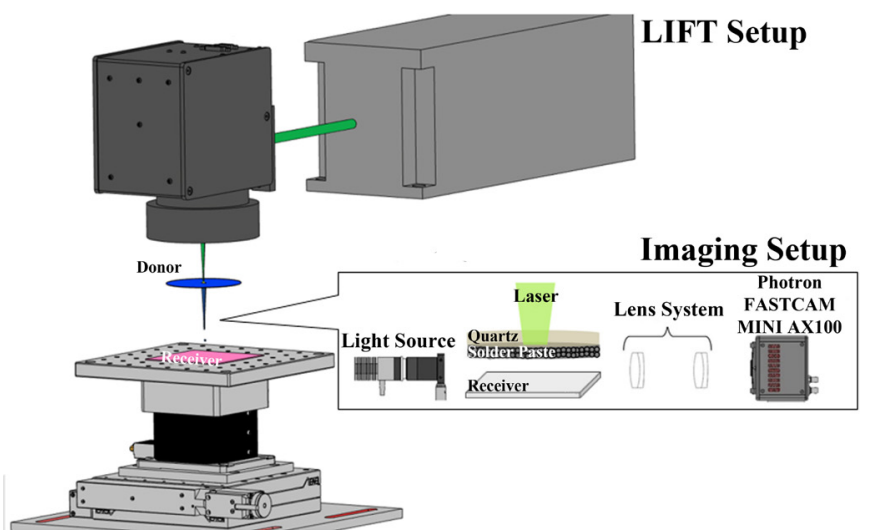

Fig.1 Schematic representation of LIFT and imaging setup

\subsection{Solder paste material}

Solder paste was purchased from Alpha Assembly Solutions. It is a lead-free, no-clean solder paste designed for use in Jet Printers. The material's properties are listed in Table 1.

Table 1 Solder paste properties

\begin{tabular}{|c|c|c|c|c|}
\hline $\begin{array}{l}\text { Solder } \\
\text { Paste }\end{array}$ & Type & $\begin{array}{l}\text { Particle } \\
\text { size }\end{array}$ & Category & Flux \\
\hline $\begin{array}{l}\text { ALPHA } \\
\text { JP-500 }\end{array}$ & $\underset{5}{\text { Type }}$ & $\begin{array}{c}15-25 \\
\mu \mathrm{m}\end{array}$ & $\begin{array}{c}\text { SAC305 } \\
96.5 \% \text { tin } \\
3 \% \text { silver } \\
0.5 \% \text { copper }\end{array}$ & $\begin{array}{l}\text { Zero halogen } \\
\text { flux formula- } \\
\text { tion, colorless } \\
\text { flux residue }\end{array}$ \\
\hline
\end{tabular}

\subsection{Donor preparation and Receiver substrate}

Two different kinds of donor substrates were prepared in order to investigate ejection mechanism and deposition of the paste. For Donor 1, no dynamic release layer (DRL) was utilized, and solder paste layer was coated $(30 \mu \mathrm{m}$ 
thick) using doctor blade film application on the donor substrate (Quartz Window, 50mm dia x 3mm thick purchased from UQG Optics). For Donor 2, solder paste layer was coated (30 $\mu \mathrm{m}$ thick) on Ag NP ink used as DRL. The DRL was prepared by spin coating Ag NP ink (SunChemicals, 20 wt.\% silver content) onto the donor substrate at 1000 rpm for 30 seconds. The Ag NP ink is then dried at $75^{\circ} \mathrm{C}$ for 10 minutes and sintered at $175^{\circ} \mathrm{C}$ for 10 minutes resulting in a thin (300 nm thick) Ag layer. [17] Silver is chosen as the DRL for compatibility reasons, since the solder paste already includes a small percentage of silver.

The quartz windows donor substrates involved in this study have very high transmission at $532 \mathrm{~nm}(>95 \%)$ and very low surface roughness $(<2 \mathrm{~nm})$. The receiving substrate was Silicon substrate.

\section{Results and discussion}

The main goal of this work was to investigate the capability of LIFT to print solder paste patterns with micrometer sized features in a controllable and consistent manner. Although LIFT can directly transfer a vast variety of materials, several demonstrations have been enabled by the presence of a DRL. In this respect the current section will begin with a comparison of the results obtained from the use of the two types of donors described in section 2.3 (with and without DRL). Other key- parameters for this investigation are the donor receiver gap and the laser fluence which can define both the form factors and the quality of the printed bumps. The optimization of these parameters for specific types of receiving substrates enables the printing of reproducible arrays of bumps which will be presented in the following section.

\subsection{Effect of DRL}

Figure 2 shows still images acquired from videos recorded (at $127.500 \mathrm{fps}$ ) by using the high-speed camera visualization setup. Initially, we studied solder paste transfer without the use of a DRL (Donor 1), as shown in Figure 2a. It was observed that a voxel of solder paste separates from the donor and travels towards the receiver substrate landing after $50 \mu$ s as a printed bump. On the contrary, in Figure $2 b$, visualizing DRL assisted LIFT, a jet is formed and reaches the receiver after $15 \mu \mathrm{s}$. Detachment of the jet and retraction towards the donor is a long procedure lasting several hundreds $\mu \mathrm{sec}$. A printed bump is observed after $1.5 \mathrm{msec}$.

In Figure 2c the ejection velocities at laser fluences of 0.9 and $1.0 \mathrm{~J} / \mathrm{cm}^{2}$ and 1.8 and $2.4 \mathrm{~J} / \mathrm{cm}^{2}$ are presented for the DRL assisted and for DRL-free LIFT respectively. Ejection velocity refers to the average jet front velocity. More specifically, the velocity was calculated by a linear fit of the propagation length of the jet front before impact depending on the elapsed time for each successive frame extracted from the videos. As expected, the velocities increase as the laser fluence increases. Furthermore, DRL assisted LIFT results in higher ejection velocities (up to $4.5 \mathrm{~m} / \mathrm{s}$ ) with respect to the DRL-free case which exhibits higher laser fluence ejection threshold. Therefore, in order to use low laser fluence and have smoother printing results (i.e. more reproducible, homogeneous and geometrically defined deposits), in this work we opted for DRL-assisted LIFT.
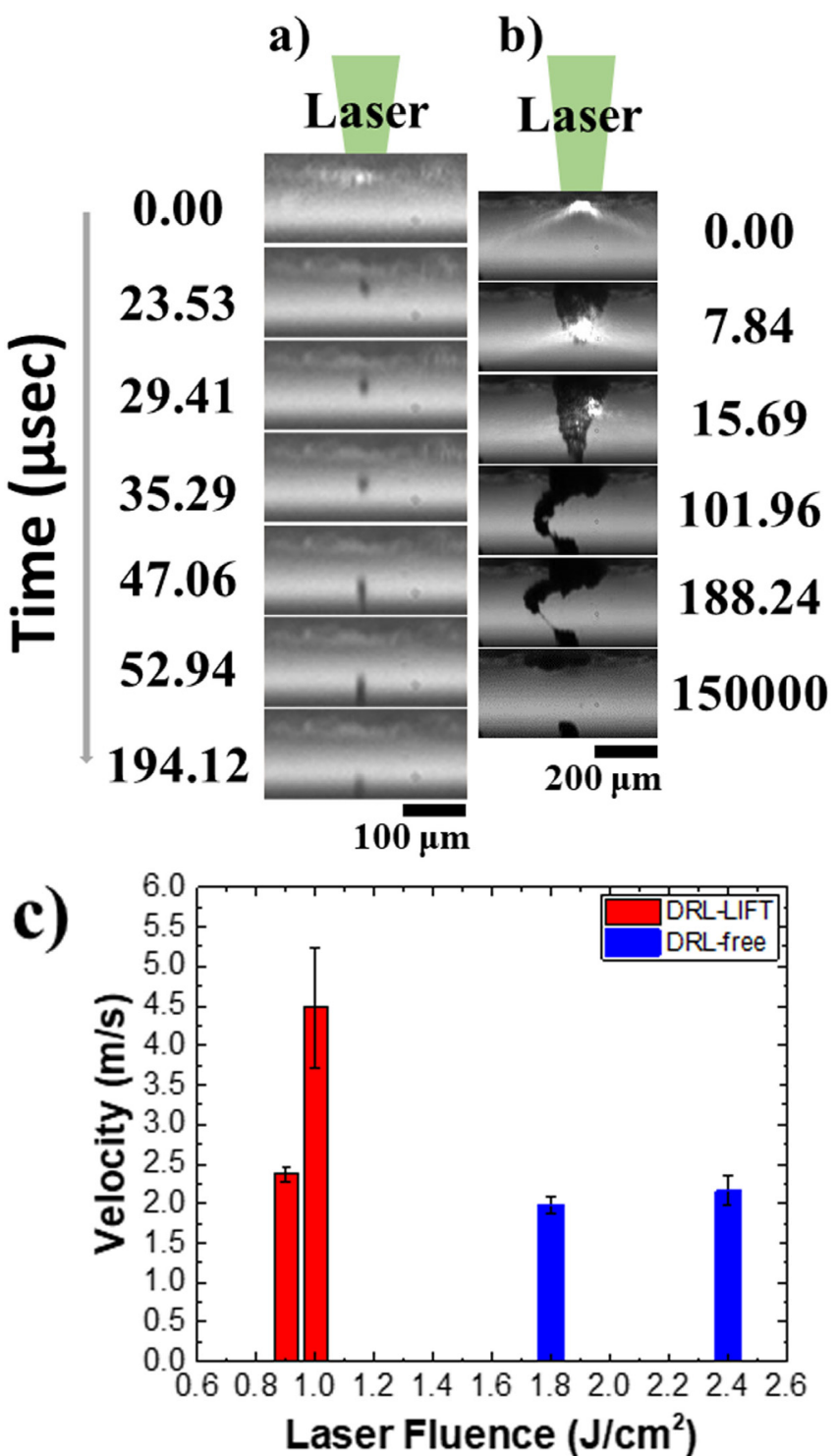

Fig. 2 LIFT of solder paste (a) without a DRL, (b) with a metallic DRL formed from a low viscous Ag NP ink. (c) Ejection velocity for two different laser fluences 0.9 and $1.0 \mathrm{~J} / \mathrm{cm}^{2}$, and 1.8 and $2.4 \mathrm{~J} / \mathrm{cm}^{2}$ for DRL-LIFT and DRL-free, respectively.

\subsection{Effect of donor/receiver gap}

In Figure 3a, when donor/receiver gap is $100 \mu \mathrm{m}$, it is observed that solder paste reaches the receiver substrate while still connected to the donor, forming a liquid bridge wetting both substrates. The pillar shaped bridge gradually decreases in width with time and after $3 \mathrm{msec}$ separates into two pillars. The pillar breaks after $>150 \mathrm{msec}$ leaving one central and one smaller printed solder bump on the receiver. In Figure $3 b$, when donor/receiver gap is wider $(200 \mu \mathrm{m})$, it is observed that a directional jet is formed (containing the metal microparticles) and when its tip reaches the receiver a small part detaches leaving a printed solder bump. In the latter case ejection and printing mechanism is much faster taking place for about $1.5 \mathrm{~ms}$. In Figure $3 \mathrm{c}$, the printed bump volume is correlated to the laser fluence for two different donor/ receiver gaps. For the $200 \mu \mathrm{m}$ gap case, an almost exponential increase of the printed volume is observed, whereas for the smaller gap of $100 \mu \mathrm{m}$, the printed volume shows a linear dependence on the laser fluence. The latter case will be presented in the next section. 
a)
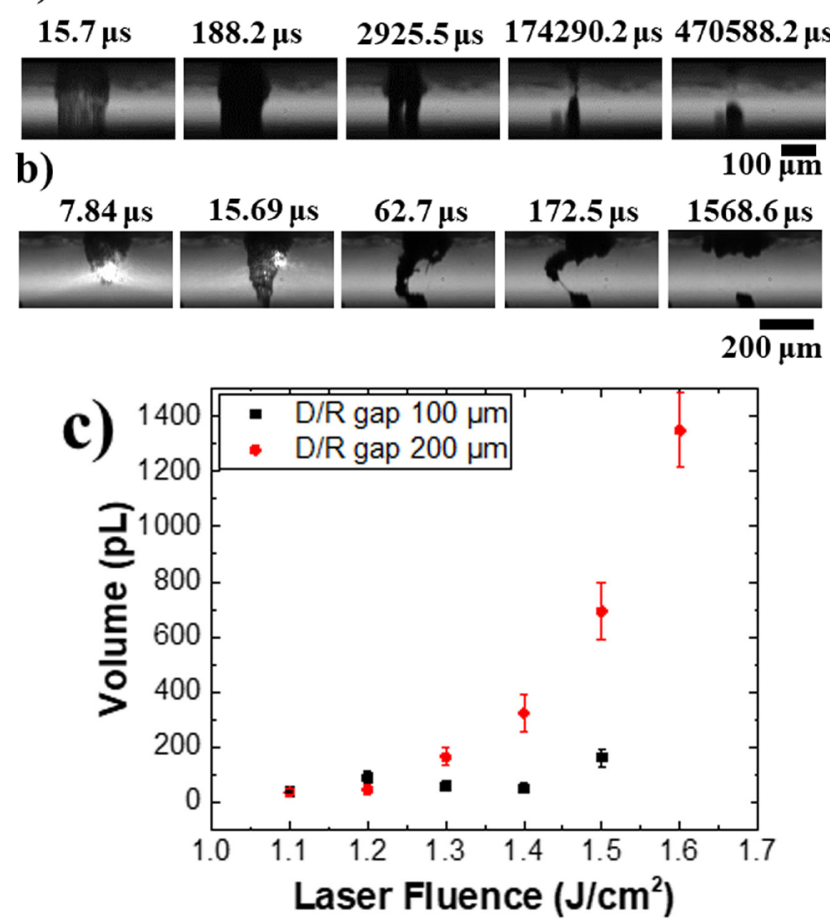

Fig. 3 DRL assisted LIFT of solder paste varying donor/receiver gap: a) $100 \mu \mathrm{m}$; b) $200 \mu \mathrm{m}$. c) Printed volume of the solder bumps as a function of laser fluence for two different donor/ receiver gaps $100 \mu \mathrm{m}$ black square; $200 \mu \mathrm{m}$ red circle

\subsection{Effect of laser fluence}

The parametric investigation is of major value for each LIFT process: laser fluence values lower than the transfer threshold result in no -or incomplete transfer, whereas very high fluences compromise the reproducibility and inflict fragmentation of the printed bump and increased debris. Figure 4 summarizes the dependence of the printed bumps form factors (height and diameter) as a function of laser fluence, for Donor 2 (with DRL). For increasing laser fluence both the height and the diameter of the bumps increase. From this figure it is evident that the threshold value is $1 \mathrm{~J} / \mathrm{cm}^{2}$, with which a bump height is comparable with the diameter of a single microparticle. Therefore, lower fluences do not allow bump transfer, whereas fluences higher than $1.4 \mathrm{~J} / \mathrm{cm}^{2}$ result in large variation of the bumps size and shape. In addition, LIFT printing of solder paste for higher fluences $\left(>1.4 \mathrm{~J} / \mathrm{cm}^{2}\right)$ tends to be more explosive resulting in multiple printed bumps, hence, repeatability and accuracy cannot always be achieved for higher fluences.

Figure 5 shows a SEM of printed solder bumps at 1.1 and $1.2 \mathrm{~J} / \mathrm{cm}^{2}$. Some residue from the solder flux is visible, and transferred solder microparticles tend to accumulate in the central region of the transferred bump.

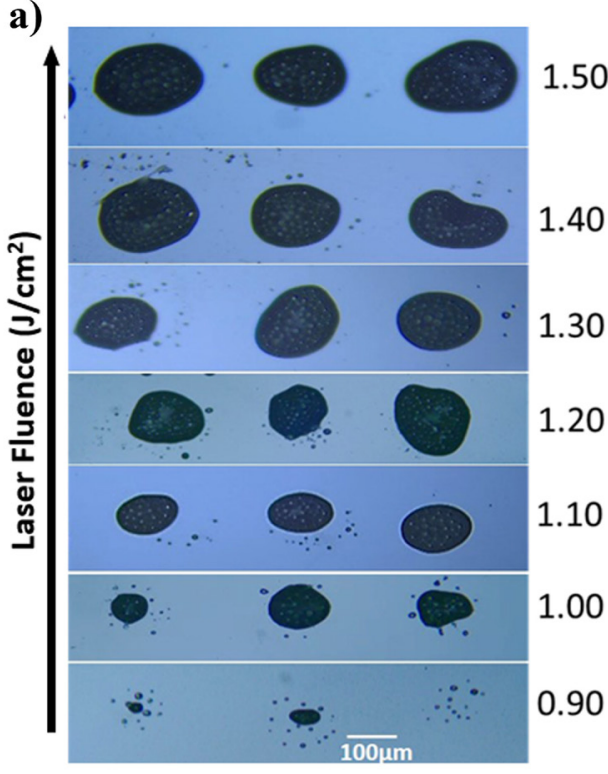

b)

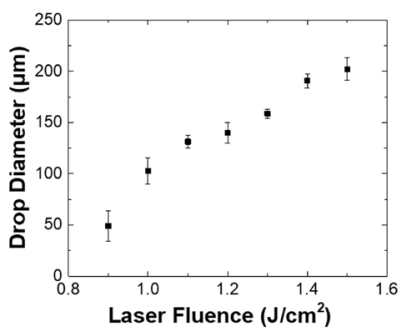

c)

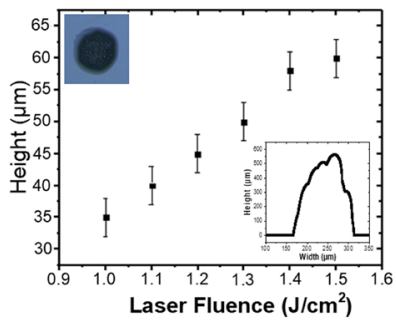

Fig. 4 a) Optical microscope images of LIFT printed solder bumps with increasing laser fluences. b) Printed diameter as a function of laser fluence, c) Printed bump height as a function of laser fluence.

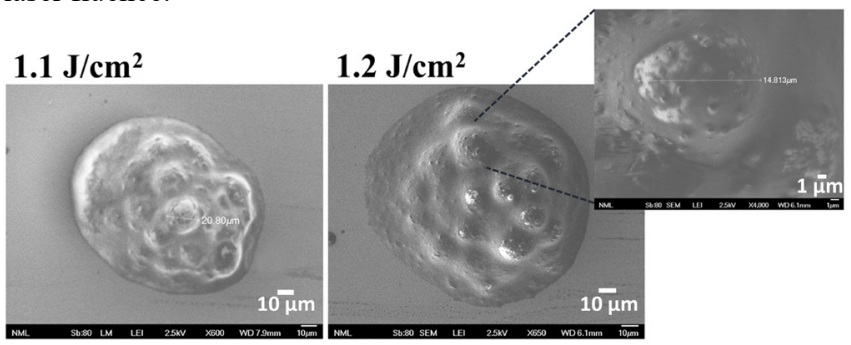

Fig. 5 SEM image showing solder morphology of printed bumps at 1.1 and $1.2 \mathrm{~J} / \mathrm{cm}^{2}$, respectively.

The max repetition rate leading to satisfactory results in this work was $100 \mathrm{kHz}$. Using a repetition rate in the range of $10-100 \mathrm{kHz}$, the reproducible printing of large arrays of bumps has been achieved. The reported results comprise arrays with no missing spots or overlapping spots which would lead to short circuits, therefore the yield in terms of printing is $100 \%$. However, taking into consideration some ill -shaped deposits and the relatively limited number of printed arrays for the industrial standards, one can estimate a yield of $>95 \%$ using LIFT under optimal printing parameters.

\subsection{Printing of solder paste lines}

In Figure 6 an optical microscopy image is shown of printed bumps at different spacing between consecutive pulses. Spacing is defined as the center-to-center distance between two consecutive pulses. More specifically, at spacing larger than $250 \mu \mathrm{m}$, isolated bumps are printed onto the Si sub- 
strate. By decreasing the spacing to $200 \mu \mathrm{m}$, the merging of neighboring bumps can be observed. The optimal printing spacing for printing a continuous line is found for a spacing ranging between 100 and $150 \mu \mathrm{m}$, were line uniformity is maintained over a distance of $850 \mu \mathrm{m}$ with a width of 160 $\mu \mathrm{m}$. The laser operated at $10 \mathrm{kHz}$, and laser fluence was fixed at $1.2 \mathrm{~J} / \mathrm{cm}^{2}$.

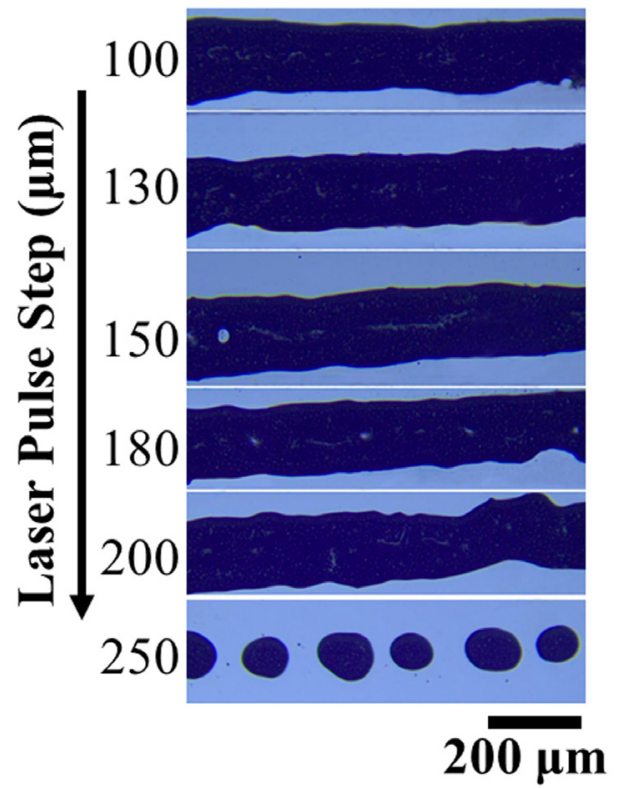

Fig. 6 Image of printed bumps at different spacing between consecutive pulses on Si substrate.

Following the laser printing of lines, oven curing at $245^{\circ} \mathrm{C}$, which is the indicated temperature of reflow soldering was carried out. The post-curing resulting structures showed ohmic behaviour.

\section{Conclusions}

The high-speed laser printing of patterns comprising solder paste has been investigated in this work. The compatibility of LIFT with solder paste for high resolution chip bonding applications has been demonstrated. In particular, the effects of DRL, donor/receiver gap and laser fluence have been thoroughly studied in order to conclude on the optimal parameters for the specific process and materials. The involvement of a $300 \mathrm{~nm}$ Ag DRL facilitates the ejection of controllable jets; a donor -to- receiver gap of less than $100 \mu \mathrm{m}$ yields the most reproducible printing results; the laser fluence threshold for these parameters (DRL and $100 \mu \mathrm{m}$ gap) is $1 \mathrm{~J} / \mathrm{cm}^{2}$, and the printed bump dimensions (min feature size of $50 \mu \mathrm{m}$ ) increase with increasing fluence until roughly $1.4 \mathrm{~J} / \mathrm{cm}^{2}$, at which point the printing becomes non-controllable and non-reproducible. The reported results validate the compatibility of LIFT with soldering processes such as flip chip bonding, offering a digital, high-resolution and high-speed analogue to standard methods such as stencil printing. The reported results set a milestone towards the incorporation of LIFT in microelectronic packaging and assembly technology, as the constantly increasing laser processing speed will soon enable throughputs compatible with large scale organic electronic manufacturing tools.

\section{Acknowledgments}

This research has been co - financed by the European Union and Greek national funds through the Operational Program Competitiveness, Entrepreneurship and Innovation, under the call RESEARCH-CREATE-INNOVATE (project code: T1EDK-00814).

\section{References}

[1] D. Wojciechowski, J. Vanfleteren, E. Reese and H.-W. Hagedorn: Microelectron. Reliab., 40, (2000), 1215.

[2] M. Zou, Y. Ma, X. Yuan, Y. Hu, J. Liu, and Z. Jin: J. Semicond. 39, (2018), 011010.

[3] D. Lu, Y. Grace Li, C. Wong: J. Adhes. Sci. Technol., 22, (2008), 815.

[4] M. P. Y. Desmulliez, R. W. Kay, S. Stoyanov and C. Bailey: Proc. 6th Electronics Packaging Technology Conf. (EPTC) (2004), 354.

[5] R. S. Braudy: Proc. IEEE, 57, (1969), 1771

[6] M. L. Levene, R. D. Scott, B. W. Siryj: Appl. Opt. 9, (1970), 2260.

[7] J. Bohandy, B. Kim, F. J. Adrian: J. Appl. Phys., 60, (1986), 1538.

[8] Ph. Delaporte, I. Zergioti, D. Karnakis: Laser processing of flexible organic electronic materials, Chapter 12 in the Handbook of Flexible Organic Electronics, S. Logothetidis (ed.), Woodhead Publishing, (2014).

[9] D. Munoz-Martina, C.F. Brasz, Y. Chena, M. Morales, C.B. Arnold and C. Molpeceres: Appl. Surf. Sci., 366, (2016), 389.

[10] C. Boutopoulos, I. Kalpyris, E. Serpetzoglou, and I. Zergioti: Microfluidics and Nanofluidics 16, (2013), 493.

[11] C. B. Arnold, P. Serra, and A. Pique: MRS Bulletin 32, (2007), 23.

[12] R. C. Y. Auyeung, H. Kim, S. A. Mathews and A. Piqué: J. Laser Micro/ Nanoeng. 2, (2007), 103.

[13] F. Zacharatos, M. Makrygianni, R. Geremia, E. Biver, D. Karnakis, S. Leyder, D. Puerto, P. Delaporte and I. Zergioti: Appl. Surf. Sci. 374, (2016), 117.

[14] S. Papazoglou, M. Makrygianni, I. Zergioti, M. Filippidou and S. Chatzandroulis: Proc. in 2016 IEEE SENSORS, Orlando, FL, (2016), pp. 1.

[15] K. Andritsos, I. Theodorakos, F. Zacharatos and I. Zergioti: App. Surf. Sci., 506, (2020) 144968.

[16] Y. Chen, D. Munoz-Martin, M. Morales, C. Molpeceres E. Sánchez-Cortezon, and J. Murillo-Gutierrez: Phys. Proc. 83, (2016), 204.

[17] S. A. Mathews, N. A. Charipar, R. C.Y. Auyeung, H. Kim and A. Piqué: Proc. SPIE, Vol. 9351, Laser-based Micro- and Nanoprocessing IX, (2015), 93510Y.

[18] K. S. Kaur, J. Missinne, B. Vandecasteele, G. Van Steenberge, S. M. Perinchery, E. C. P. Smits, R. Mandamparabil, Proc. 2013 Conf. on Lasers \& Electro-Optics Europe \& International Quantum Electronics Conference CLEO EUROPE/IQEC, Munich, (2013), pp. 1.

[19] I. Theodorakos, A. Kalaitzis, M. Makrygianni, A. Hatziapostolou, A Kabla, S. Melamed, F. de la Vega and I. Zergioti: Adv. Eng. Mater., 21, (2019) 1900605.

(Received: June 27, 2020, Accepted: October 13, 2020) 